\title{
RECOVERY OF HIGH FREQUENCY WAVE FIELDS FOR THE ACOUSTIC WAVE EQUATION
}

\author{
HAILIANG LIU AND JAMES RALSTON
}

\begin{abstract}
Computation of high frequency solutions to wave equations is important in many applications, and notoriously difficult in resolving wave oscillations. Gaussian beams are asymptotically valid high frequency solutions concentrated on a single curve through the physical domain, and superposition of Gaussian beams provides a powerful tool to generate more general high frequency solutions to PDEs. An alternative way to compute Gaussian beam components such as phase, amplitude and Hessian of the phase, is to capture them in phase space by solving Liouville type equations on uniform grids. Following [3] we present a systematic construction of asymptotic high frequency wave fields from computations in phase space for acoustic wave equations; the superposition of phase space based Gaussian beams over two moving domains is shown necessary. Moreover, we prove that the $k$-th order Gaussian beam superposition converges to the original wave field in the energy norm, at the rate of $\epsilon^{\frac{k}{2}+\frac{1-n}{4}}$ in dimension $n$.
\end{abstract}

\section{Contents}

1. Introduction

2. Phase space based Gaussian beam Ansatz

3. Recovery of the high frequency wave fields

4. Control of initial error

5. Propagation of the approximation error

6. An example

7. Higher order Approximations

Acknowledgments

References

\section{INTRODUCTION}

This is the continuation of our project, initiated in [3], of developing a rigorous recovery theory for high frequency wave fields from phase space based computations. Here we focus on the wave equation

$$
P u:=\left[\partial_{t}^{2}-c(x)^{2} \Delta\right] u=0, \quad(x, t) \in \mathbb{R}^{n} \times \mathbb{R},
$$

where $c(x)$ is a positive smooth function, with highly oscillatory initial data

$$
u(x, 0)=A_{\text {in }}(x, \epsilon) e^{i S_{\text {in }}(x) / \epsilon}, \quad u_{t}(x, 0)=B_{\text {in }}(x, \epsilon) e^{i S_{\text {in }}(x) / \epsilon} .
$$

Date: June 15, 2009.

Key words and phrases. High frequency waves, Gaussian beams, phase space, superposition. 
The initial phase $S_{\text {in }} \in C^{\infty}\left(\mathbb{R}^{n}\right)$, and the amplitudes $A_{\text {in }}, B_{\text {in }} \in C_{0}^{\infty}\left(\mathbb{R}^{n}\right)$ have the following asymptotic expansions:

$$
\begin{aligned}
& A_{\text {in }}:=A_{\text {in }}^{(0)}(x)+\epsilon A_{\text {in }}^{(1)}(x)+\epsilon^{2} A_{\text {in }}^{(2)}(x)+\cdots, \\
& B_{\text {in }}:=\epsilon^{-1} B_{\text {in }}^{(-1)}(x)+B_{\text {in }}^{(0)}(x)+\epsilon B_{\text {in }}^{(1)}(x)+\cdots .
\end{aligned}
$$

The small parameter $\epsilon$ represents the typical wave length of oscillations of the initial data. Propagation of oscillations of wave length $\epsilon$ causes mathematical and numerical challenges in solving high frequency wave propagation problems.

In this article we are interested in the construction of globally valid asymptotic wave fields and the analysis of their convergence to the true solutions of the initial value problem. A general discussion of this problem and background references are given in the introduction to [3]. We have two objectives:

i) to present the construction of asymptotic solutions as superpositions over phase space;

ii) to estimate the difference between the exact wave fields and the asymptotic ones.

The construction for (i) is based on Gaussian beams (GB) in physical space constructed similarly to those given for wave equations in [6], but here the construction is carried out by solving inhomogeneous Liouville equations in phase space. While the result is no longer a superposition of asymptotic solutions to the wave equation (1.1), the superposition is nonetheless asymptotic. We consider superpositions over two subdomains moving with two Hamiltonian flows, respectively, and show that they are asymptotic solutions by relating them to the Lagrangian superposition through two time-dependent symplectic changes of variables. An argument of this type was used for the Helmholtz equation in [2].

For (ii), as in [6], we use the well-posedness theory for (1.1), i.e. the continuous dependence of solutions of $P \psi=f$ on their initial data and $f$. Thus, the sources of error in the Gaussian beam superposition for the initial value problem are the error in approximating the initial data and the error in solving the PDE. There are some differences between the acoustic wave equation and the Schrödinger wave equation. For example, the caustics that can form are weaker.

In summary, our phase space based Gaussian beam superposition is expressed as

$$
u^{\epsilon}(t, y)=Z(n, \epsilon)\left[\int_{\Omega^{+}(t)} u_{P G B}^{+}(t, y, X) d X+\int_{\Omega^{-}(t)} u_{P G B}^{-}(t, y, X) d X\right],
$$

where $X=(x, p)$ denotes variables in phase space $\mathbb{R}^{2 n}, \Omega(0)$ is the domain where we construct initial Gaussian beams from the given data, and $\Omega^{ \pm}(t)$ is the image of $\Omega(0)$ under the Hamiltonian flow for $H(x, p)= \pm c(x)|p|$. The functions $u_{P G B}^{ \pm}(t, y, X)$ are constructed using the phase space based Gaussian beam Ansatz, and $Z(n, \epsilon) \sim \epsilon^{-n / 2}$ is a normalization parameter. Our result shows that for the $k$-th order phase space Gaussian beam superposition, the following estimate holds

$$
\left\|\left(u^{\epsilon}-u\right)(t, \cdot)\right\|_{E} \lesssim \|\left(u^{\epsilon}(0, \cdot)-u_{\text {in }}(\cdot) \|_{E}+|\Omega(0)| \epsilon^{\frac{k}{2}+\frac{1-n}{4}},\right.
$$

where $\|e\|_{E}^{2}:=\frac{\epsilon^{2}}{2} \int_{\mathbb{R}^{n}}\left[c^{-2}\left|e_{t}\right|^{2}+\left|\nabla_{x} e\right|^{2}\right] d x$. Here and in what follows we use $A \lesssim B$ to denote the estimate $A \leq C B$ for a constant $C$ which is independent of $\epsilon$. 
For the initial data of the form $\left(A_{\text {in }}(x, \epsilon), B_{\text {in }}(x, \epsilon)\right) e^{i S_{\text {in }}(x) / \epsilon}$ we need a superposition over an n-dimensional submanifold of phase space. The asymptotic solution is then represented as

$$
u^{\epsilon}(t, y)=Z(n, \epsilon)\left[\int_{\Omega^{+}(t)} u_{P G B}^{+} \delta\left(w^{+}\right) d X+\int_{\Omega^{-}(t)} u_{P G B}^{-} \delta\left(w^{-}\right) d X\right],
$$

where $w^{ \pm}$is obtained from the Liouville equation

$$
\partial_{t} w+H_{p} \cdot \nabla_{x} w-H_{x} \cdot \nabla_{p} w=0, \quad w(0, X)=p-\nabla_{x} S_{\text {in }}(x),
$$

with $H(x, p)= \pm c(x)|p|$, respectively. Our result shows that

$$
\left\|\left(u^{\epsilon}-u\right)(t, \cdot)\right\|_{E} \lesssim \epsilon^{\frac{k}{2}+\frac{1-n}{4}} .
$$

Here the exponent $k / 2$ reflects the accuracy of the Gaussian beam in solving the PDE. It will increase when one uses more accurate beams. The exponent $\frac{1-n}{4}$ indicates the damage done by the caustics.

We now conclude this section by outlining the rest of this paper: in Section 2 we start with Gaussian beam solutions in physical space, and define the phase space based GB Ansatz through the Hamiltonian map. Section 3 is devoted to a recovery scheme through superpositions over two moving domains. The total error is shown to be bounded by an initial error and the evolution error of order $\epsilon^{(3-n) / 4}$. Control of initial error is discussed in Section 4. Convergence rates are obtained for first order GB solutions in Section 5. In Section 6 we present an example to illustrate these constructions. Extensions to higher order GB approximations are given in Section 7 .

\section{Phase space Based Gaussian beam Ansatz}

As is well known, the idea underlying Gaussian beams [5] is to build asymptotic solutions concentrated on a single ray path in $\mathbb{R}_{t} \times \mathbb{R}_{x}^{n}$. This means that, given a ray path $\gamma$ parameterized by $(t, x(t))$, one makes the ansatz

$$
u^{\epsilon}(t, y)=A(t, y, \epsilon) e^{i \Phi(t, y) / \epsilon},
$$

where $\Phi(t, x(t))$ is real, and $\operatorname{Im}\{\Phi(t, y)\}>0$ for $y \neq x(t)$. The amplitude is allowed to be complex and has an asymptotic expansion in terms of $\epsilon$ :

$$
A(t, y, \epsilon)=A_{0}(t, y)+\epsilon A_{1}(t, y)+\cdots+\epsilon^{N} A_{N}(t, y) \text {. }
$$

We wish to build asymptotic solutions to $P u(t, y)=0$, i.e., we want $P u^{\epsilon}=O\left(\epsilon^{M}\right)$. Substituting from (2.1) and grouping terms multiplied by the same power of $\epsilon$, we obtain the equations of geometric optics:

$$
P\left[A(t, y, \epsilon) e^{i \Phi(t, y) / \epsilon}\right]=\left(\sum_{j=-2}^{N} c_{j}(t, y) \epsilon^{j}\right) e^{i \Phi(t, y) / \epsilon},
$$

where for $G(t, y)=\left|\partial_{t} \Phi\right|^{2}-c^{2}\left|\nabla_{y} \Phi\right|^{2}$,

$$
\begin{aligned}
c_{-2}(t, y) & =-G(t, y) A_{0}, \\
c_{-1}(t, y) & =2 i L A_{0}+G(t, y) A_{1}, \\
c_{l-1}(t, y) & =2 i L A_{l}+G(t, y) A_{l+1}+P\left[A_{l-1}\right], \quad l=1, \cdots N-1 .
\end{aligned}
$$


Here $L$ is the linear differential operator,

$$
L=\Phi_{t} \partial_{t}-c^{2} \nabla_{y} \Phi \cdot \nabla_{y}+\frac{1}{2} P[\Phi] .
$$

Since $e^{i \Phi / \epsilon}$ decays rapidly away from $\gamma$, to make $P\left(A e^{i \Phi / \epsilon}\right)=O\left(\epsilon^{M}\right)$ for a given $M \in \mathbb{Z}$, we only need to make $c_{j}$ vanish on $\gamma$ to sufficiently high order. In this work we discuss mainly the lowest order Gaussian beam solutions, followed by an extension to higher order Gaussian beam superpositions in Section 7.

We begin with $c_{-2}=0$, i.e. $G=0$. This leads to two eikonal equations

$$
\partial_{t} \Phi+H\left(x, \nabla_{x} \Phi\right)=0, \quad H(x, p)= \pm c(x)|p| .
$$

The leading amplitude solves

$$
\partial_{t} A+H_{p} \cdot \nabla_{x} A=\frac{A P[\Phi]}{2 H\left(x, \nabla_{x} \Phi\right)} .
$$

We continue to denote the phase space variable as $X=(x, p)$, and let $X_{0}=\left(x_{0}, p_{0}\right)$ denote the initial state. Then the equations for the bicharacteristics $X=X^{ \pm}\left(t, X_{0}\right)$ originating from $X_{0}$ at $t=0$ are

$$
\frac{d}{d t} X\left(t, X_{0}\right)=V\left(X\left(t, X_{0}\right)\right), \quad X\left(0, X_{0}\right)=X_{0} .
$$

The vector field $V=\left(H_{p},-H_{x}\right)$ is divergence free, and hence this flow preserves the volume on phase space.

From now on we include the initial data $X_{0}$ as a parameter in the phase: $\Phi=$ $\Phi\left(t, y ; X_{0}\right)$ and the amplitude: $A=A\left(t, y ; X_{0}\right)$. We apply Taylor expansion of the phase $\Phi$ and the amplitude $A$ about $x=x\left(t, X_{0}\right)$ to obtain

$\Phi\left(t, y ; X_{0}\right)=S\left(t ; X_{0}\right)+p\left(t, X_{0}\right)\left(y-x\left(t, X_{0}\right)\right)+\frac{1}{2}\left(y-x\left(t, X_{0}\right)\right)^{\top} M\left(t ; X_{0}\right)\left(y-x\left(t, X_{0}\right)\right)$, with $p\left(t, X_{0}\right)=\partial_{y} \Phi\left(t, x\left(t, X_{0}\right) ; X_{0}\right)$ and

$$
S\left(t ; X_{0}\right)=\Phi\left(t, x\left(t, X_{0}\right) ; X_{0}\right), \quad M\left(t ; X_{0}\right)=\partial_{y}^{2} \Phi\left(t, x\left(t, X_{0}\right) ; X_{0}\right) .
$$

For the amplitude we set $A\left(t, y ; X_{0}\right)=A\left(t ; X_{0}\right)$ with $A\left(t ; X_{0}\right)=A\left(t, x\left(t, X_{0}\right) ; X_{0}\right)$. Then we get the equations along the curve $\gamma$ for $S$

$$
\frac{d}{d t} S\left(t ; X_{0}\right)=0, \quad S\left(0 ; X_{0}\right)=S_{\text {in }}\left(x_{0}\right)
$$

and the Hessian $M$

$$
\frac{d}{d t} M\left(t ; X_{0}\right)+H_{x x}+H_{x p} M+M H_{p x}+M H_{p p} M=0, \quad M\left(0 ; X_{0}\right)=M_{\text {in }}\left(x_{0}\right) .
$$

Using the eikonal equation $\partial_{t} \Phi+H(x, \nabla \Phi)=0$ twice, we see that

$$
P[\Phi]=\partial_{t}[-H(x, \nabla \Phi)]-c^{2} \Delta \Phi=H_{p} \cdot H_{x}+H_{p} M H_{p}-c^{2} \operatorname{Tr}(M) .
$$

This with (2.4) shows that the amplitude along the ray, $A\left(t ; X_{0}\right)$, satisfies

$$
\frac{d}{d t} A\left(t ; X_{0}\right)=\frac{A}{2 H}\left[H_{p} \cdot H_{x}+H_{p} M H_{p}-c^{2} \operatorname{Tr}(M)\right], \quad A\left(0 ; X_{0}\right)=A_{\text {in }}\left(x_{0}\right) .
$$

We have introduced this form of the transport equation because it is easier to translate to Eulerian coordinates. The essential idea behind the Gaussian beam method 
is to choose some complex Hessian $M_{\text {in }}$ initially so that $M$ remains bounded for all time, and its imaginary part is positive definite. Equation (2.9) shows that the amplitude $A\left(t ; X_{0}\right)$ will also remain bounded for all time.

The above construction ensures that the following GB Ansatz is an approximate solution

$$
u_{G B}\left(t, y ; X_{0}\right)=u_{G B}^{+}\left(t, y ; X_{0}\right)+u_{G B}^{-}\left(t, y ; X_{0}\right)
$$

where

$$
u_{G B}^{ \pm}\left(t, y ; X_{0}\right)=A^{ \pm}\left(t ; X_{0}\right) \exp \left(\frac{i}{\epsilon} \Phi^{ \pm}\left(t, y ; X_{0}\right)\right),
$$

where both $A^{ \pm}\left(t ; X_{0}\right)$ and $\Phi^{ \pm}\left(t, y ; X_{0}\right)$ are computed from (2.9) and (2.6) with $H=$ $\pm c(x)|p|$, respectively.

Here $A^{ \pm}(0 ; X)$ are to be chosen so that a superposition will match the initial data

$$
\left.\left(u, u_{t}\right)\right|_{t=0}=\left(A_{\text {in }}, B_{\text {in }}\right) e^{i S_{\text {in }} / \epsilon}
$$

to leading order. For this matching we need (for $X=\left(x, \nabla S_{\text {in }}(x)\right)$ )

$$
\begin{aligned}
& A^{+}(0 ; X)+A^{-}(0 ; X)=A_{\mathrm{in}}^{(0)}(x), \\
& \frac{i}{\epsilon} A^{+}(0 ; X) \partial_{t} \Phi^{+}(0, x ; X)+\frac{i}{\epsilon} A^{-}(0 ; X) \partial_{t} \Phi^{-}(0, x ; X)=\frac{1}{\epsilon} B_{\mathrm{in}}^{(-1)}(x) .
\end{aligned}
$$

In the second relation we took only the leading term in $e^{-i S_{\mathrm{in}} / \epsilon} u_{t}$. Since the two Hamiltonians have different signs,

$$
\Phi^{ \pm}(0, x ; X)=S_{\text {in }}(x) \quad \text { and } \quad \partial_{t} \Phi^{ \pm}(0, x ; X)=\mp c(x)\left|\nabla_{x} S_{\text {in }}(x)\right|,
$$

the second relation gives

$$
A^{+}(0 ; X)-A^{-}(0 ; X)=\frac{i B_{\text {in }}^{(-1)}(x)}{c(x)\left|\nabla_{x} S_{\text {in }}(x)\right|} .
$$

Hence solving for $A^{ \pm}$we have

$$
A^{ \pm}(0 ; X)=\frac{1}{2}\left(A_{\text {in }}^{(0)}(x) \pm \frac{i B_{\text {in }}^{(-1)}(x)}{c(x)\left|\nabla_{x} S_{\text {in }}(x)\right|}\right) .
$$

Note that we could simplify the superposition by taking some special initial data such that $B_{\text {in }}^{(-1)}(x)=-i A_{\text {in }}^{(0)}(x) c(x)\left|\nabla S_{\text {in }}(x)\right|$. The advantage of these special choices is that we do not need a sum of two Gaussians to approximate the solution. We also note that for given initial $B_{\text {in }}^{\epsilon}$ of order $O(1)$, i.e., $B_{\text {in }}^{(-1)}=0$, we see that $A^{ \pm}(0 ; X)=$ $\frac{1}{2} A_{\text {in }}^{(0)}(x)$.

\section{RECOVERY OF THE HIGH FREQUENCY WAVE FIELDS}

Since the wave equation we consider is linear, the high frequency wave field $u$ at $(t, y)$ in physical space is expected to be generated by a superposition of neighboring Gaussian beams

$$
u^{\epsilon}(t, y)=Z(n, \epsilon) \int_{\Omega(0)} u_{G B}\left(t, y ; X_{0}\right) d X_{0}
$$


where $\Omega(0)$ is a bounded open set containing

$$
\left\{X_{0}: \quad x_{0} \in \operatorname{supp}\left(A_{\text {in }}\right) \cup \operatorname{supp}\left(B_{\text {in }}\right), \quad p_{0} \in \operatorname{range}\left(\partial_{x} S_{\text {in }}\right)\right\} .
$$

The normalization parameter $Z(n, \epsilon) \sim \epsilon^{-n / 2}$ is determined by matching initial data against the Gaussian profile.

Since the flows $X^{ \pm}\left(t ; X_{0}\right)$ are volume preserving in phase space,

$$
\operatorname{det}\left(\frac{\partial X^{ \pm}\left(t, X_{0}\right)}{\partial X_{0}}\right)=1
$$

Using $X=X^{ \pm}\left(t, X_{0}\right)$ and their inverses $X_{0}=X_{0}^{ \pm}(t, X)$, we obtain our Gaussian beam Ansatz in phase space

$$
u_{P G B}^{ \pm}(t, y, X):=u_{G B}^{ \pm}\left(t, y ; X_{0}^{ \pm}(t, X)\right) .
$$

From (3.1) it follows that

$$
\begin{aligned}
u^{\epsilon}(t, y) & =Z(n, \epsilon) \int_{\Omega(0)}\left[u_{G B}^{+}\left(t, y ; X_{0}\right)+u_{G B}^{-}\left(t, y ; X_{0}\right)\right] d X_{0} \\
& =Z(n, \epsilon)\left[\int_{\Omega^{+}(t)} u_{P G B}^{+}(t, y, X) d X+\int_{\Omega^{-}(t)} u_{P G B}^{-}(t, y, X) d X\right]
\end{aligned}
$$

where

$$
\Omega^{ \pm}(t)=X^{ \pm}(t, \Omega(0))
$$

Each phase space Gaussian beam has the form

$$
u_{P G B}(t, y, X)=\tilde{A}(t, X) \exp \left(\frac{i}{\epsilon} \tilde{\Phi}(t, y, X)\right)
$$

where

$$
\Phi(t, y, X)=\tilde{S}(t, X)+p \cdot(y-x)+\frac{1}{2}(y-x)^{\top} \tilde{M}(t, X)(y-x) .
$$

Note that though $u_{P G B}^{ \pm}(t, y, X)$ are no longer asymptotic solutions of the wave equation in $(t, y)$, their superpositions over the moving domains $\Omega^{ \pm}(t)$ in $X$ remain asymptotic solutions.

Let $\mathcal{L}$ be the Liouville operator defined by

$$
\mathcal{L}:=\partial_{t}+V \cdot \nabla_{X}
$$

If $\tilde{w}(t, X)$ is the phase space representative of $w\left(t ; X_{0}\right)$ in the sense that $w\left(t ; X_{0}\right)=$ $w\left(t, X\left(t, X_{0}\right)\right)$ for any $t>0$, then

$$
\frac{d}{d t} w\left(t ; X_{0}\right)=\mathcal{L} \tilde{w}(t, X) .
$$

Hence from the Lagrangian formulation of equations for $(S, M, A)$ we obtain PDEs for $(\tilde{S}, \tilde{M}, \tilde{A})$ in (2.7), (2.8) and (2.9) :

$$
\begin{aligned}
& \mathcal{L}(\tilde{S})=0, \quad \tilde{S}(0, X)=S_{\mathrm{in}}(x), \\
& \mathcal{L}(\tilde{M})+H_{x x}+H_{x p} \tilde{M}+\tilde{M} H_{p x}+\tilde{M} H_{p p} \tilde{M}=0, \quad \tilde{M}(0, X)=M_{\mathrm{in}}(x), \\
& \mathcal{L}(\tilde{A})=\frac{\tilde{A}}{2 H}\left[H_{p} \cdot H_{x}+H_{p} \tilde{M} H_{p}-c^{2} \operatorname{Tr}(\tilde{M})\right], \quad \tilde{A}(0, X)=A_{\text {in }}(x),
\end{aligned}
$$


where $H(x, p)=c(x)|p|$ or $H(x, p)=-c(x)|p|$. The heart of the matter is equation (3.7). It is known from [4] that, if $M_{\text {in }}$ is symmetric and the imaginary part of $M_{\text {in }}$ is positive definite, then a global solution $\tilde{M}$ to (3.7) is guaranteed and has the properties:

i) $\tilde{M}=\tilde{M}^{T}$, and

ii) $\operatorname{Im}(\tilde{M})$ is positive definite for all $t>0$.

There are several ways of computing $\tilde{M}$. Following [1] (see also [3, Section 7]), we use a level set method to construct the Hessian:

$$
\tilde{M}=-g_{x}\left(g_{p}\right)^{-1}
$$

where $g=\phi_{1}(t, X)+i \phi_{2}(t, X)$ with $\phi_{i}$ obtained by solving the Liouville equation

$$
\mathcal{L}(\phi)=0 .
$$

From the well-posedness theory of the wave equation we have the following.

Lemma 3.1. Let $u$ satisfy $P[u]=0$ in $[0, T] \times \mathbb{R}^{n}$ with $\left(u, u_{t}\right)$ given at $t=0$, and let $u^{\epsilon}$ be an asymptotic solution. Then the error $e=u^{\epsilon}-u$ satisfies

$$
\|e(t)\|_{E} \leq\|e(0)\|_{E}+\epsilon \int_{0}^{t}\left\|c^{-1} P\left[u^{\epsilon}\right]\right\|_{L^{2}} d \tau,
$$

where $\|e\|_{E}=\sqrt{2 E}$ and

$$
E:=\frac{\epsilon^{2}}{2} \int_{\mathbb{R}^{n}}\left[c^{-2}\left|e_{t}\right|^{2}+\left|\nabla_{x} e\right|^{2}\right] d x .
$$

Proof. Since we start with the data with compact support, at any finite time the support of the solution remains bounded (due to finite speed of propagation for the wave equation).

Let $e=u^{\epsilon}-u$. Then from $P[u]=0$

$$
P[e]=P\left[u^{\epsilon}\right]-P[u]=P\left[u^{\epsilon}\right] .
$$

We now have

$$
\begin{aligned}
\frac{d}{d t} E(t) & =\epsilon^{2} \int_{R^{n}}\left[c^{-2} e_{t} e_{t t}+\nabla e \cdot \nabla e_{t}\right] d x \\
& =\epsilon^{2} \int_{R^{n}}\left[\nabla \cdot\left(e_{t} \nabla e\right)+c^{-2} e_{t} P\left[u^{\epsilon}\right]\right] d x \\
& \leq \epsilon^{2}\left\|c^{-1} e_{t}\right\|_{L^{2}}\left\|c^{-1} P\left[u^{\epsilon}\right]\right\|_{L^{2}} \leq \epsilon \sqrt{2 E}\left\|c^{-1} P\left[u^{\epsilon}\right]\right\|_{L^{2}}
\end{aligned}
$$

This, upon integration in time, leads to the desired estimate.

\section{Control of initial ERror}

For the initial phase $S_{\text {in }}$, we set $p_{0}=\nabla_{x} S_{\text {in }}\left(x_{0}\right)$ and form the Lagrangian superpositions

$$
u^{\epsilon}(t, y)=Z(n, \epsilon) \int_{\Omega(0)} u_{G B}\left(t, y ; X_{0}\right) \delta\left(p_{0}-\nabla_{x} S_{\text {in }}\left(x_{0}\right)\right) d X_{0} .
$$


In order to track the deformation of the surface $p-\nabla_{x} S_{\text {in }}(x)=0$ as time evolves, we introduce two level set functions $w=w^{ \pm}(t, X)$ such that

$$
\mathcal{L}[w]=0, \quad w(0, X)=p-\nabla_{x} S_{\text {in }}(x),
$$

with $H(x, p)= \pm c(x)|p|$. Here $w^{ \pm}$gives $\phi_{2}$ needed in (3.9) and $\phi_{1}$ can be obtained from solving the respective Liouville equation with $\phi_{1}(0, X)=x$.

Using the volume preserving maps $X=X^{ \pm}\left(t, X_{0}\right)$, leads to the Gaussian beam superposition in phase space

$$
u^{\epsilon}(t, y)=Z(n, \epsilon)\left[\int_{\Omega^{+}(t)} u_{P G B}^{+} \delta\left(w^{+}\right) d X+\int_{\Omega^{-}(t)} u_{P G B}^{-} \delta\left(w^{-}\right) d X\right],
$$

where $\Omega^{ \pm}(t)=X^{ \pm}(t, \Omega(0))$. Our choice of initial data for the beams in this superposition will be made to match the initial data in (1.2). Set

$$
I(0)=\left\{x: \quad(x, p) \in \Omega(0), \quad p=\nabla_{x} S_{\text {in }}(x)\right\} .
$$

We now use the Lagrangian formulation of the GB superposition to match the initial data.

$$
u^{\epsilon}(t, y)=Z(n, \epsilon) \int_{I(0)} u_{G B}\left(t, y ; x_{0}\right) d x_{0}
$$

Here and in what follows we use $u_{G B}\left(t, y ; x_{0}\right)$ for $u_{G B}\left(t, y ; x_{0}, \nabla_{x} S_{\text {in }}\left(x_{0}\right)\right)$. If we take $S^{ \pm}\left(0 ; x_{0}\right)=S_{\text {in }}\left(x_{0}\right), M^{ \pm}\left(0 ; x_{0}\right)=\partial_{x}^{2} S_{\text {in }}\left(x_{0}\right)+i \beta I$ with $\beta>0$ as well as $A^{ \pm}\left(0 ; X_{0}\right)$ as defined in (2.11), then

$$
u^{\epsilon}(0, y)=Z(n, \epsilon) \int_{I(0)} A_{\text {in }}^{(0)}\left(x_{0}\right) e^{i \Phi\left(0, y ; x_{0}\right) / \epsilon} d x_{0}
$$

where

$$
\Phi\left(0, y ; x_{0}\right)=T_{2}^{x_{0}}\left[S_{\text {in }}\right](y)-\frac{\beta}{2}\left|y-x_{0}\right|^{2} .
$$

Here $T_{j}^{x}[S](y)$ denotes the $j^{\text {th }}$ order Taylor polynomial of $S$ about $x$ at the point $y$. Setting

$$
Z(n, \epsilon)=\left(\frac{\beta}{2 \pi \epsilon}\right)^{n / 2}
$$

we have

$$
u^{\epsilon}(0, y)=\int_{I(0)} A_{\text {in }}^{(0)}\left(x_{0}\right) e^{\frac{i}{\epsilon}\left[T_{2}^{x_{0}}\left[S_{\mathrm{in}}\right](y)\right]} K\left(x_{0}-y, \frac{\epsilon}{2 \beta}\right) d x_{0},
$$

where $K(x, \tau)=\frac{1}{(4 \pi \tau)^{n / 2}} e^{-\frac{|x|^{2}}{4 \tau}}$ is the usual heat kernel, satisfying $\operatorname{limit}_{\tau \downarrow 0} K(x, \tau)=$ $\delta(x)$ as distributions on $\mathbb{R}^{n}$, and

$$
\int_{x} K(x-y, \tau) d x=1, \quad \forall \tau>0, y \in \mathbb{R}^{n} .
$$

On the other hand the initial wave field is

$$
u(0, y)=A_{\text {in }}^{\epsilon}(y) e^{i S_{\text {in }}(y) / \epsilon}=\int_{\mathbb{R}^{n}} A_{\text {in }}^{\epsilon}(y) e^{i S_{\text {in }}(y) / \epsilon} K\left(x-y, \frac{\epsilon}{2 \beta}\right) d x .
$$


Both the phase and amplitude in the integrand can be approximated by their Taylor expansion when $|x-y|$ is small, say $|x-y|<\epsilon^{1 / 3}$, and the integral over the complement of this neighborhood will then be $O\left(\exp \left(-c \epsilon^{-1 / 3}\right)\right)$ for some $c>0$. Thus the main contributions to the error come from the remainder terms in the Taylor expansions, and this leads to

Lemma 4.1. 6] Let $S_{\text {in }} \in C^{\infty}\left(R^{n}\right)$ be a real-valued function, and $A_{\text {in }} \in C_{0}^{\infty}\left(R^{n}\right)$. Then

$$
\begin{aligned}
\left\|u(0, \cdot)-u^{\epsilon}(0, \cdot)\right\|_{L^{2}} & \lesssim \epsilon^{\frac{1}{2}}, \\
\epsilon\left\|u(0, \cdot)-u^{\epsilon}(0, \cdot)\right\|_{H^{1}} & \lesssim \epsilon^{\frac{1}{2}} .
\end{aligned}
$$

Remark 4.1. We note that a cutoff function is necessary and important when one is building beams of higher accuracy

We now show the initial error of time derivative of the GB superposition is also under control. We compute the time derivative of (4.4) to obtain

$$
\partial_{t} u^{\epsilon}(t, y)=Z(n, \epsilon) \int_{I(0)} \partial_{t} u_{G B}\left(t, y ; x_{0}\right) d x_{0},
$$

where $u_{G B}\left(t, y ; x_{0}\right)=u_{G B}^{+}\left(t, y ; x_{0}\right)+u_{G B}^{-}\left(t, y ; x_{0}\right)$ with

$$
\partial_{t} u_{G B}^{ \pm}\left(t, y ; x_{0}\right)=\left[\partial_{t} A^{ \pm}+\frac{i}{\epsilon} A^{ \pm} \partial_{t} \Phi^{ \pm}\right] e^{i \Phi^{ \pm}\left(t, y ; x_{0}\right) / \epsilon} .
$$

Note that the GB construction ensures that

$$
\partial_{t} \Phi^{ \pm}\left(t, y ; x_{0}\right)=\mp c(y)\left|\nabla \Phi^{ \pm}\left(t, y ; x_{0}\right)\right|+O\left(\left|y-x\left(t, x_{0}\right)\right|^{3}\right) .
$$

Recall (2.9) we have $\partial_{t} A\left(t ; x_{0}\right) \sim O(1)$. Hence from (2.10) we have

$$
\partial_{t} u_{G B}\left(0, y ; x_{0}\right)=\left[O(1)+\epsilon^{-1}\left(B^{(-1)}\left(x_{0}\right)+O\left(\left|y-x_{0}\right|\right)\right)\right] e^{i \Phi^{ \pm}\left(0, y ; x_{0}\right) / \epsilon} .
$$

Note that

$$
\left\|Z(n, \epsilon) \int_{I(0)}\left[O(1)+O\left(\frac{\left|y-x_{0}\right|}{\epsilon}\right)\right] e^{i \Phi^{ \pm}\left(0, y ; x_{0}\right) / \epsilon} d x_{0}\right\|_{L_{y}^{2}} \leq C\left(1+\epsilon^{-1 / 2}\right),
$$

which together with Lemma 4.1 again gives

$$
\left\|u^{\epsilon}(0, \cdot)-u(0, \cdot)\right\|_{E} \leq \epsilon\left\|u^{\epsilon}(0, \cdot)-u(0, \cdot)\right\|_{H^{1}}+\epsilon\left\|\partial_{t} u^{\epsilon}(0, \cdot)-\partial_{t} u(0, \cdot)\right\|_{L_{y}^{2}} \lesssim \epsilon^{1 / 2} .
$$

Remark 4.2. The above analysis shows that one could choose $B_{\text {in }}^{(-1)}$ to simplify the superposition, as was pointed out in Section 2. For example,

(i) for $B_{\text {in }}^{(-1)}=-i c(x)\left|\nabla S_{\text {in }}\right|$, then $A^{+}\left(0 ; X_{0}\right)=A_{\text {in }}^{(0)}\left(x_{0}\right), \quad A^{-}\left(0 ; X_{0}\right)=0$

$$
u^{\epsilon}(t, y)=Z(n, \epsilon)\left[\int_{\Omega^{+}(t)} u_{P G B}^{+} \delta\left(w^{+}\right) d X\right] ;
$$

(ii) for $B_{\text {in }}^{(-1)}=0$, then $A^{ \pm}\left(0 ; X_{0}\right)=\frac{1}{2} A_{\text {in }}^{(0)}\left(x_{0}\right)$

$$
u^{\epsilon}(t, y)=Z(n, \epsilon)\left[\int_{\Omega^{+}(t)} u_{P G B}^{+} \delta\left(w^{+}\right) d X+\int_{\Omega^{-}(t)} u_{P G B}^{-} \delta\left(w^{-}\right) d X\right] .
$$




\section{Propagation of the APproximation ERror}

We now turn to quantify the evolution error $P\left[u^{\epsilon}\right]$. Recall the Schur's lemma: If $[T f](y)=\int K(x, y) f(x) d x$ and

$$
\sup _{x} \int_{y}|K(x, y)| d y=C_{1}, \sup _{y} \int_{x}|K(x, y)| d x=C_{2},
$$

then

$$
\|T f\|_{L^{2}} \leq \sqrt{C_{1} C_{2}}\|f\|_{L^{2}}
$$

Proof. We have by Schwartz

$$
\begin{aligned}
|[T f](y)|^{2} \leq\left(\int|K(x, y)| f(x) d x\right)^{2} & \leq \int|K(x, y)| d x \int|K(x, y)||f(x)|^{2} d x \\
& \leq C_{2} \int|K(x, y)||f(x)|^{2} d x .
\end{aligned}
$$

So integrating both sides in $y$ and taking the square root gives the result.

We now apply Schur's lemma to a typical term in $\int_{I(0)} P\left[u^{\epsilon}\right] d x_{0}$ :

$$
[T A](y)=\int_{I(0)} A\left(t ; x_{0}\right) F\left(t, y ; x_{0}\right) e^{i \Phi\left(t, y ; x_{0}\right) / \epsilon} d x_{0},
$$

where the imaginary part of $\Phi\left(t, y ; x_{0}\right)$ is bounded below by $c I$ and for convenience we will assume that $|F| \leq\left|y-x\left(t, x_{0}\right)\right|^{k}$. Then one can apply Schur's lemma with

$$
C_{1}=\sup _{x_{0}} \int_{\mathbb{R}^{n}}\left|y-x\left(t, x_{0}\right)\right|^{k} e^{-(c / \epsilon)\left|y-x\left(t, x_{0}\right)\right|^{2}} d y=\epsilon^{\frac{k}{2}+\frac{n}{2}} \int_{z}|z|^{k} e^{-c|z|^{2}} d z, \quad \text { and }
$$

$$
C_{2}(t, \epsilon)=\sup _{y} \int_{I(0)}\left|y-x\left(t, x_{0}\right)\right|^{k} e^{-(c / \epsilon)\left|y-x\left(t, x_{0}\right)\right|^{2}} d x_{0} .
$$

In general one does not know what $C_{2}(t, \epsilon)$ will be. As long as $A$ has compact support $C_{2}$ will be at least bounded by $c \epsilon^{k / 2}$. Thus the error in $L^{2}$ norm will be bounded by $c \epsilon^{k / 2+n / 4}$. We now show that for the wave equation, a better rate can be obtained.

Lemma 5.1. We have

$$
C_{2}(t, \epsilon) \lesssim \epsilon^{(k+1) / 2}
$$

Proof. From (2.7) and taking $p_{0}=\nabla_{x} S_{\text {in }}\left(x_{0}\right)$ it follows

$$
S\left(t, x\left(t, x_{0}\right)\right)=S_{\text {in }}\left(x_{0}\right), \quad \forall t>0 .
$$

Differentiation of this equation in $x_{0}$ gives

$$
\frac{\partial x}{\partial x_{0}} p=p_{0}, \quad p\left(t, x_{0}\right):=\nabla_{x} S\left(t, x\left(t, x_{0}\right)\right) .
$$

For non-constant initial phase, at least one element in the deformation matrix $\frac{\partial x}{\partial x_{0}}$ is non-zero. Assume $\frac{\partial x_{1}}{\partial x_{01}} \neq 0$ near $x_{0}^{*}$, then writing $x_{0}=\left(x_{01}, \hat{x}_{0}\right)$ there exists a function $h$ such that $x_{01}=h\left(t, z, \hat{x}_{0}\right)$ and

$$
z \equiv x_{1}\left(t, h\left(t, z, \hat{x}_{0}\right), \hat{x}_{0}\right)
$$


in the neighborhood of $x_{0}^{*}$. Also the map $\left(x_{01}=h\left(t, z, \hat{x}_{0}\right), \hat{x}_{0}\right) \rightarrow\left(z, \hat{x}_{0}\right)$ is invertible, with the Jacobian determined by

$$
J=\operatorname{det}\left(\frac{\partial\left(x_{01}, \hat{x}_{0}\right)}{\partial\left(z, \hat{x}_{0}\right)}\right)=\left|\frac{\partial h}{\partial z}\right|=\left|\frac{\partial x_{1}}{\partial x_{01}}\right|^{-1}
$$

With this map we rewrite the underlying quantity as

$$
C_{2}=\int_{\left(z, \hat{x}_{0}\right)}\left(\left|\hat{y}-\hat{x}\left(t, z, \hat{x}_{0}\right)\right|^{2}+\left|y_{1}-z\right|^{2}\right)^{k / 2} \exp \left(-\frac{c}{\epsilon}\left(\left|\hat{y}-\hat{x}\left(t, z, \hat{x}_{0}\right)\right|^{2}+\left|y_{1}-z\right|^{2}\right)\right) J d \hat{x}_{0} d z
$$

Using a stretched coordinate in $z$ so that $z-y_{1}=\sqrt{\epsilon} \xi$, with $a:=\hat{y}-\hat{x}\left(t, z, \hat{x}_{0}\right)$, we obtain

$$
C_{2}=\sqrt{\epsilon} \int_{\left(\xi, \hat{x}_{0}\right)}\left(|a|^{2}+\epsilon|\xi|^{2}\right)^{k / 2} e^{-c|\xi|^{2}} \exp \left(-\frac{c}{\epsilon}|a|^{2}\right) J d \hat{x}_{0} d \xi
$$

Rewriting $e^{-c|\xi|^{2}}=e^{-c|\xi|^{2} / 2} \cdot e^{-c|\xi|^{2} / 2}$, and using the fact that $e^{-c|\xi|^{2} / 2} \leq 1$ and $|\xi|^{2} e^{-c|\xi|^{2} / 2} \leq C$, we obtain

$$
\begin{aligned}
C_{2} & \leq \sqrt{\epsilon} \int_{\left(\xi, \hat{x}_{0}\right)}\left(|a|^{2}+C \epsilon\right)^{k / 2} e^{-c|\xi|^{2} / 2} e^{-c|a|^{2} / \epsilon} J d \hat{x}_{0} d \xi \\
& \lesssim \sqrt{\epsilon} \epsilon^{k / 2} \int_{\left(\xi, \hat{x}_{0}\right)} e^{-c|\xi|^{2} / 2} J d \hat{x}_{0} d \xi
\end{aligned}
$$

Here we have used the fact that $\left(|a|^{2}+C \epsilon\right)^{k / 2} e^{-c|a|^{2} / \epsilon} \lesssim \epsilon^{k / 2}$ for any $a \in \mathbb{R}^{n-1}$. As long as the initial domain for $x_{0}$ is finitely compact, the above integral is uniformly bounded. Note that the local feature of the used map is not restricted, since one could use a partition of unity to decompose $C_{2}$ into a finite sum of terms with the same rate of error. The desired estimate thus follows.

This lemma enables us to conclude the following key estimate

$$
\|T[A]\|_{L^{2}} \lesssim \epsilon^{k / 2+(1+n) / 4}
$$

which will be used to prove the following theorem.

Theorem 5.2. Let $P=\partial_{t}^{2}-c^{2}(x) \Delta$ be the linear wave operator and $u^{\epsilon}$ be defined in (4.2) with $\operatorname{Im}\left(M_{\mathrm{in}}^{ \pm}\right)=\beta I$ and $Z(n, \epsilon)=(\beta /(2 \pi \epsilon))^{n / 2}$. If both $A_{\mathrm{in}}$ and $B_{\mathrm{in}}$ have compact supports, then $u^{\epsilon}$ is an asymptotic solution and satisfies

$$
\left\|P\left[u^{\epsilon}\right](t, \cdot)\right\|_{L_{y}^{2}} \lesssim \epsilon^{-\frac{1+n}{4}}
$$


Proof. Using the volume-preserving map of $X=X\left(t, X_{0}\right)$ and $w\left(t, X\left(t, X_{0}\right)\right)=$ $w\left(0, X_{0}\right)$, we obtain

$$
\begin{aligned}
u^{\epsilon}(t, y) & =Z(n, \epsilon) \int_{\Omega(0)} u_{P G B}\left(t, y, X\left(t, X_{0}\right)\right) \delta\left(w\left(t, X\left(t, X_{0}\right)\right)\right) d X_{0} \\
& =Z(n, \epsilon) \int_{\Omega(0)} u_{G B}\left(t, y ; X_{0}\right) \delta\left(w\left(0, X_{0}\right)\right) d X_{0} \\
& =Z(n, \epsilon) \int_{\Omega(0)} u_{G B}\left(t, y ; X_{0}\right) \delta\left(p_{0}-\nabla_{x} S_{\mathrm{in}}\left(x_{0}\right)\right) d X_{0} \\
& =Z(n, \epsilon) \int_{I(0)} u_{G B}\left(t, y ; x_{0}\right) d x_{0} .
\end{aligned}
$$

According to the GB construction, $u_{G B}\left(t, y ; x_{0}\right)$ is an asymptotic solution for each $x_{0}$, so will be their superpositions $u^{\epsilon}(t, y)$. It remains to verify (5.3). First we see that

$$
P\left[u^{\epsilon}(t, y)\right]=Z(n, \epsilon) \int_{I(0)} P\left[u_{G B}\left(t, y ; x_{0}\right)\right] d x_{0},
$$

where

$$
P\left[A\left(t ; x_{0}\right) e^{i \Phi\left(t, y ; x_{0}\right) / \epsilon}\right]=\left(\epsilon^{-2} c_{-2}(t, y)+\epsilon^{-1} c_{-1}+c_{0}\right) e^{i \Phi\left(t, y ; x_{0}\right) / \epsilon},
$$

where for $G(t, y)=\left|\partial_{t} \Phi\right|^{2}-c^{2}\left|\nabla_{y} \Phi\right|^{2}$, we have

$$
\begin{aligned}
c_{-2}(t, y) & =-G(t, y) A, \\
c_{-1}(t, y) & =2 i\left[\partial_{t} A \partial_{t} \Phi+\frac{1}{2} A P[\Phi]\right], \\
c_{0}(t, y) & =\partial_{t}^{2} A\left(t ; x_{0}\right) .
\end{aligned}
$$

Using Taylor expansion around $x=x\left(t, x_{0}\right)$ we have

$$
G(t, y)=G(t, x)+\partial_{x} G(t, x) \cdot(y-x)+\frac{1}{2}(y-x)^{\top} \partial_{x}^{2} G(y-x)+O\left(|y-x|^{3}\right) .
$$

Then the Gaussian beam construction sketched in Section 2 ensures that

$$
\left|c_{-2}(t, y)\right| \leq C|A||y-x|^{3} .
$$

Also using the construction for $A$, we are able to show

$$
\left|c_{-1}(t, y)\right| \leq C|A||y-x|, \quad\left|c_{0}(t, y)\right| \leq C|A| .
$$

The construction with positive $\operatorname{Im}(M)$ guarantees that

$$
\Phi\left(t, y ; x_{0}\right) \geq c|y-x|^{2} .
$$

Consequently,

$$
\begin{aligned}
Z^{-1}\left\|P\left[u^{\epsilon}(t, \cdot)\right]\right\|_{L^{2}} & \leq\left\|\int_{I(0)} A e^{-I m\left(\Phi\left(t, y ; x_{0}\right)\right) / \epsilon}\left|\epsilon^{-2} c_{-2}+\epsilon^{-1} c_{-1}+c_{0}\right| d x_{0}\right\|_{L_{y}^{2}} \\
& \leq \sum_{j=-2}^{0} \epsilon^{j}\left\|\int_{I(0)}|A|\left|c_{j}\right| e^{-c\left|y-x\left(t, x_{0}\right)\right|^{2} / \epsilon} d x_{0}\right\|_{L_{y}^{2}},
\end{aligned}
$$


continuing the estimate by using the key estimate (5.2) with $k=3,1,0$ for $F=$ $c_{-2}, c_{-1}, c_{0}$, respectively

$$
\begin{aligned}
& \lesssim\left[\epsilon^{-2} \epsilon^{3 / 2}+\epsilon^{-1} \cdot \epsilon^{1 / 2}+1\right] \epsilon^{(1+n) / 4} \\
& \lesssim \epsilon^{-1 / 2+(1+n) / 4}
\end{aligned}
$$

which when using $Z \sim \epsilon^{-n / 2}$ proves the result.

This combined with the obtained initial error and total error estimate in Lemma 3.1 gives

Theorem 5.3. Given $T>0$, and let $u$ be the solution of the wave equation subject to the initial data $\left(u, u_{t}\right)(0, x)=\left(A^{\epsilon}, B_{\text {in }}\right) e^{i S_{\mathrm{in}}(x) / \epsilon}$. Let $u^{\epsilon}$ be the first order approximation defined in (4.2) with initial data satisfying $S^{ \pm}(0 ; x)=S_{\mathrm{in}}(x), M^{ \pm}(0 ; x)=$ $\partial_{x}^{2} S_{\mathrm{in}}(x)+i \beta I$, and $A^{ \pm}(0 ; x)=\frac{1}{2}\left(A_{\mathrm{in}}^{(0)}(x) \pm \frac{i B_{\mathrm{in}}^{(-1)}}{c(x)\left|\nabla_{x} S_{\mathrm{in}}\right|}\right)$ with $\left|\operatorname{supp}\left(A_{\mathrm{in}}^{\epsilon}\right)\right|+\left|\operatorname{supp}\left(B_{\mathrm{in}}^{\epsilon}\right)\right|<$ $\infty$. Then there exists $\epsilon_{0}>0$, a normalization parameter $Z(n, \epsilon)=\left(\frac{\beta}{2 \pi \epsilon}\right)^{n / 2}$, and a constant $C$ such that for all $\epsilon \in\left(0, \epsilon_{0}\right)$

$$
\left\|\left(u^{\epsilon}-u\right)(t, \cdot)\right\|_{E} \leq C \epsilon^{\frac{1}{2}+\frac{1-n}{4}}
$$

for $t \in[0, T]$.

\section{An EXAMPLE}

Consider the initial value problem in $\mathbb{R}^{3}$ for $\partial_{t}^{2} u-\Delta u=0$ with initial data

$$
u(0, x)=e^{i|x| / \epsilon} \frac{f(|x|)}{|x|} \text {, and } u_{t}(0, x)=0,
$$

where $f(s) \in C_{0}^{\infty}(0, \infty)$. Setting $g(s)=f(s) \exp (i s / \epsilon)$ for $s>0$, we extend $g(s)$ to be odd on $\mathbb{R}$, i.e.

$$
g(-|x|)=-f(|x|) \exp (i|x| / \epsilon)
$$

This problem has the exact solution

$$
u(t, x)=\frac{1}{|x|}(g(t+|x|)-g(t-|x|)) .
$$

At $x=0$ this solution has a caustic of the maximum possible strength, since all rays starting inward from the sphere $|x|=a$ arrive at $x=0$ when $t=a$. This is reflected in the behavior of the exact solution

$$
u(0, t)=g^{\prime}(t)=\left(i f(t) / \epsilon+f^{\prime}(t)\right) e^{i t / \epsilon},
$$

which grows like $\epsilon^{-1}$ as $\epsilon$ goes to zero.

To build a Gaussian beam approximation for this we need

$$
u_{G B}(t, x)=\frac{1}{2}\left(\frac{\beta}{2 \pi \epsilon}\right)^{3 / 2} \int_{\mathbb{R}^{3}} A^{+}(t, y) e^{i \Phi^{+}(t, x ; y) / \epsilon}+A^{-}(t, y) e^{i \Phi^{-}(t, x ; y) / \epsilon} d y,
$$

where $A^{ \pm}(0, y)=f(|y|) /|y|$ and

$$
\Phi^{ \pm}(0, x ; y)=|y|+(x-y) \cdot p(y)+(x-y) \cdot\left(\frac{1}{|y|}(I-P(y)+i \beta I)(x-y) / 2,\right.
$$


where $p(y)=y /|y|$ and $P(y)$ is the orthogonal projection on the span of $p(y)$. We also want $\Phi_{t}^{+}(0, x ; y)=-\Phi_{t}^{-}(0, x ; y)$, so that $\partial_{t} u(0, x)=0$ and $A^{ \pm}(t, y) \exp \left(i k \Phi^{ \pm}(t, x ; y)\right)$ must be a lowest order Gaussian beams concentrated on the null bi-characteristics for $\tau \pm|\xi|$. With these definitions we have

$$
\begin{gathered}
\Phi^{ \pm}(t, x ; y)=|y|+\left(x-x^{ \pm}(t, y)\right) \cdot p(y)+ \\
\frac{1}{2}\left(x-x^{ \pm}(t, y)\right) \cdot\left[i \beta P(y)+\frac{1+i \beta|y|}{|y| \pm t(1+i \beta|y|)}(I-P(y))\right]\left(x-x^{ \pm}(t, y)\right),
\end{gathered}
$$

where $x^{ \pm}(t, x ; y)=y \pm t p(y)$. For the amplitudes we have

$$
A^{ \pm}(t, x \pm t p(y))=(1 \pm t(1+i \beta))^{-1} A(0 ; x) .
$$

Evaluating $u_{G B}(t, x)$ analytically looks difficult, but for $u_{G B}(t, 0)$ one has for $t>0$

$$
u_{G B}(t, 0)=u(t, 0)+o(1 / \epsilon) .
$$

This behavior is predicted by the basic result that, like Fourier integral operators, Gaussian beam superpositions give accurate leading order terms in asymptotic expansions.

In principle, one can evaluate the Gaussian beam superposition and compare it with the exact solution. Doing this numerically could lead to interesting results on the accuracy of these superpositions.

\section{Higher order Approximations}

The accuracy of the phase space based Gaussian beam superposition depends on accuracy of the individual Gaussian beam Ansatz. Gaussian beams can be constructed to satisfy the wave equation modulo errors of order $\epsilon^{N}$, for arbitrary $N$, by computing higher order terms in the spatial Taylor series for the phase and amplitude about the central ray. If we refer the construction in previous sections as the first order GB solution, then a $k^{\text {th }}$ order GB solution will include the Taylor series up to $(k+1)^{t h}$ order for the phase, and $(k-1-2 l)^{t h}$ order for the $l^{t h}$ amplitude $A_{l}$ for $l=0, \cdots,\left[\frac{k-1}{2}\right]$. The equations for these phase and amplitude Taylor coefficients are derived recursively, starting with the phase and then progressing through the amplitudes. At each stage (phase function, leading amplitude, next amplitude ...) one has to derive the Taylor series up to sufficiently high order before passing to the next function in the expansion.

Let $X=X^{ \pm}\left(t ; X_{0}\right)$, with $x=x^{ \pm}\left(t ; X_{0}\right)$, denote the bicharacteristic at time $t>0$, which originates from $X_{0}$. Following [6, 3] we define the $k^{\text {th }}$ order Gaussian beams as follows

$$
u_{k G B}^{ \pm}\left(t, y ; X_{0}\right)=\rho(y-x)\left[\sum_{l=0}^{\left\lfloor\frac{k-1}{2}\right\rfloor} \epsilon^{l} T_{k-1-2 l}^{x}\left[A_{l}^{ \pm}\right](y)\right] \exp \left(\frac{i}{\epsilon} T_{k+1}^{x}\left[\Phi^{ \pm}\right](y)\right),
$$

where $T_{k}^{x}[f](y)$ is the $k^{t h}$ order Taylor polynomial of $f$ about $x$ evaluated at $y$, and $\rho$ is a cut-off function such that on its support the Taylor expansion of $\Phi^{ \pm}$still has a positive imaginary part. 
By invoking the volume preserving map $X=X^{ \pm}\left(t, X_{0}\right)$ and its inverse map denoted by $X_{0}=X_{0}^{ \pm}(t, X)$, we obtain a phase space based $k^{\text {th }}$ order Gaussian beam Ansatz

$$
u_{k P G B}^{ \pm}(t, y, X):=u_{k G B}^{ \pm}\left(t, y ; X_{0}(t, X)\right)
$$

Proceeding as previously, we form the superpositions.

$$
u_{k}^{\epsilon}(t, y)=Z(n, \epsilon)\left[\int_{\Omega^{+}(t)} u_{k P G B}^{+} \delta\left(w^{+}\right) d X+\int_{\Omega^{-}(t)} u_{k P G B}^{-} \delta\left(w^{-}\right) d X\right],
$$

where $\Omega(t)=X(t, \Omega(0))$, and $w^{ \pm}(t, X)$ is the solution of the Liouville equation with $H= \pm c(x)|p|$ subject to $w^{ \pm}(0, X)=p-\nabla_{x} S_{\text {in }}(x)$.

In (7.1) the initial data for the amplitudes $A_{l}^{ \pm}$must be chosen consistently with the initial data (1.2). This leads to the recursion relations

$$
\begin{aligned}
& A_{l}^{+}(0, x)+A_{l}^{-}(0, x)=A_{\text {in }}^{(l)} \\
& \partial_{t} A_{l-1}^{+}(0, x)+\partial_{t} A_{l-1}^{-}(0, x)-i\left(A_{l}^{+}(0, x)-A_{l}^{-}(0, x)\right) c(x)\left|\nabla S_{\text {in }}(x)\right|=B_{\text {in }}^{(l-1)}(x) .
\end{aligned}
$$

Note that, since this recursion involves the initial time derivatives of the amplitudes, it becomes quite complicated as $l$ increases.

This gives a $k^{t h}$ order asymptotic solution of the wave equation. More precisely, we have the following theorem.

Theorem 7.1. Let $P$ be the linear wave operator of the form $P=\partial_{t}^{2}-c^{2} \Delta$, and $u^{\epsilon}$ is defined in (7.2) with $\operatorname{Im}\left(M_{\mathrm{in}}^{ \pm}\right)=\beta I$ and $Z(n, \epsilon)=(\beta /(2 \pi \epsilon))^{n / 2}, \beta>0$, then $u_{k}^{\epsilon}$ is an asymptotic solution and satisfies

$$
\left\|P\left[u_{k}^{\epsilon}\right](t, \cdot)\right\|_{L_{y}^{2}} \lesssim \epsilon^{\frac{k}{2}-1+\frac{1-n}{4}} .
$$

Proof. For notational convenience we estimate only one of two Gaussian beams with \pm index omitted:

$$
u_{k}^{\epsilon}(t, y)=Z(n, \epsilon) \int_{I(0)} u_{k G B}\left(t, y ; x_{0}\right) d x_{0} .
$$

According to the GB construction, $u_{k G B}\left(t, y ; x_{0}\right)$ are asymptotic solutions for each $x_{0}$, so will be their superpositions $u_{k}^{\epsilon}(t, y)$. It remains to verify (17.4). First we see that

$$
P\left[u_{k}^{\epsilon}(t, y)\right]=Z(n, \epsilon) \int_{I(0)} P\left[u_{k G B}\left(t, y ; x_{0}\right)\right] d x_{0}
$$

Using (2.2) in Section 2 with $A$ replaced by $\rho(y-x)\left[\sum_{l=0}^{\left\lfloor\frac{k-1}{2}\right\rfloor} \epsilon^{l} T_{k-1-2 l}^{x}\left[A_{l}\right](y)\right]$ and $\Phi$ by $T_{k+1}^{x}[\Phi](y)$, we have

$$
\begin{aligned}
& c_{-2}(t, y)=-\tilde{G} \rho(y-x) T_{k-1}^{x}\left[A_{0}\right](y), \\
& c_{-1}(t, y)=2 i L\left[\rho T_{k-1}^{x}\left[A_{0}\right](y)\right]+\tilde{G} T_{k-3}^{x}\left[A_{1}\right](y), \\
& \left.c_{l}(t, y)=2 i L\left[\rho T_{k-3-2 l}^{x}\left[A_{l+1}\right](y)\right]\right]+\tilde{G} \rho T_{k-5-2 l}^{x}\left[A_{l+2}\right](y)+P\left[\rho T_{k-1-2 l}^{x}\left[A_{l}\right](y)\right], \quad l=0,1, \cdots,
\end{aligned}
$$


where $\tilde{G}=\left[\left(\partial_{t} T_{k+1}^{x}[\Phi](y)\right)^{2}-c^{2}\left(\nabla_{y} T_{k+1}^{x}[\Phi](y)\right)^{2}\right]$. Using $T_{k+1}^{x}[\Phi](y)=\Phi(y)+$ $R_{k+1}^{x}[\Phi](y)$, here $R_{k+1}^{x}$ denotes the remainder of the Taylor expansion, and $\tilde{G}(t, y)=$ $O\left(|y-x|^{k+2}\right)$ we can see that

$$
\left|c_{-2}(t, y)\right| \leq C|y-x|^{k+2} \text {. }
$$

Also using the construction for $A_{l}$ and their derivatives, we are able to show

$$
\left|c_{l}(t, y)\right| \leq C|y-x|^{k-2-2 l},
$$

where we have used the fact that differentiation of $\rho$ vanishes in a neighborhood of $x$. The use of the cut-off function ensures that we can always choose a small neighborhood of $x\left(t, x_{0}\right)$ so that

$$
\operatorname{Im}\left(T_{k+1}^{x}[\Phi](y)\right) \geq c|y-x|^{2} .
$$

Consequently,

$$
\begin{aligned}
Z^{-1}\left\|P\left[u^{\epsilon}(t, \cdot)\right]\right\|_{L^{2}} & \leq\left\|\int_{I(0)} A e^{-\operatorname{Im}\left(T_{k+1}^{x}[\Phi](y)\right) / \epsilon}\left|\epsilon^{-2} c_{-2}+\epsilon^{-1} c_{-1}+c_{0}+\cdots\right| d x_{0}\right\|_{L_{y}^{2}} \\
& \leq \sum_{j=-2}^{\left\lfloor\frac{k-1}{2}\right\rfloor-2} \epsilon^{j}\left\|\int_{I(0)}|A|\left|c_{j}\right| e^{-c\left|y-x\left(t, x_{0}\right)\right|^{2} / \epsilon} d x_{0}\right\|_{L_{y}^{2}},
\end{aligned}
$$

continuing the estimate by using the key estimate (5.2)

$$
\begin{aligned}
& \lesssim\left[\epsilon^{-2} \epsilon^{k / 2+1}+\epsilon^{-1} \cdot \epsilon^{k / 2}+\cdots\right] \epsilon^{(1+n) / 4} \\
& \lesssim \epsilon^{k / 2-1+(1+n) / 4}
\end{aligned}
$$

which when using $Z \sim \epsilon^{-n / 2}$ proves the result.

In order to obtain an estimate of $\left\|\left(u_{k}^{\epsilon}-u\right)(t, \cdot)\right\|_{E}$ for any $0 \leq t \leq T$, all that remains to verify is that the superposition (4.2) accurately approximates the initial data. However, using the recursion (7.3) to determine the amplitudes, this is again an application of [6]. which shows that the initial error in energy norm is bounded by $\epsilon^{k / 2}$ for $k>1$. Thus our main result for $k^{t h}$ order phase space GB superposition is as follows.

Theorem 7.2. Given $T>0$, and let $u$ be the solution of the wave equation subject to the initial data $\left(u, u_{t}\right)(0, x)=\left(A_{\mathrm{in}}, B_{\mathrm{in}}\right) e^{i S_{\mathrm{in}}(x) / \epsilon}$, and $u^{\epsilon}$ be the $k^{\text {th }}$ order approximation defined in (7.2) with initial data chosen as described above with $\left|\operatorname{supp}\left(A_{\text {in }}\right)\right|+\left|\operatorname{supp}\left(B_{\text {in }}\right)\right|<\infty$. Then there exists $\epsilon_{0}>0$, a normalization parameter $Z(n, \epsilon) \sim \epsilon^{-n / 2}$, and a constant $C$ such that for all $\epsilon \in\left(0, \epsilon_{0}\right)$

$$
\left\|\left(u^{\epsilon}-u\right)(t, \cdot)\right\|_{E} \leq C \epsilon^{\frac{k}{2}+\frac{1-n}{4}}
$$

for $t \in[0, T]$.

\section{Remarks}

- Due to the property of symmetry in time, all results obtained apply to $|t| \leq$ $T$.

- For higher order constructions, the Liouville equation for higher order GB components can be given similarly to those for the first order GB method. 
- For computation of high order derivatives of the phase through level set functions we refer to [3] for details.

\section{ACKNOWLEDGMENTS}

Liu's research was partially supported by the National Science Foundation under the Kinetic FRG Grant DMS07-57227. He also wants to thank the IPAM for the hospitality and support during his staying for the program on "Quantum and Kinetic Transport: Analysis, Computations, and New Applications", March 9-June 12, 2009.

\section{REFERENCES}

[1] S. Jin, H. Wu, and X. Yang. Gaussian beam methods for the Schröodinger equation in the semi-classical regime: Lagrangian and Eulerian formulations. Comm. Math. Sci., 6:995-1020, 2008.

[2] S. Leung, J. Qian, and R. Burridge. Eulerian gaussian beams for highfrequency wave propagation. Geophysics, 72(5):61-76, 2007.

[3] H. Liu and J. Ralston. Recovery of high frequency wave fields from phase space based measurements. arXiv:0904.3776, 2009.

[4] J. Ralston. Gaussian beams and the propagation of singularities. Studies in PDEs, 23:206-248, 1982.

[5] J. Ralston. Gaussian beams. Notes on Gasussian beams, available at http://www.math.ucla.edu/ ralston/pub/Gaussnotes.pdf, 2005.

[6] N. M. Tanushev. Superpositions and higher order gaussian beams. Commun. Math. Sci., $6(2): 449-475,2008$.

Iowa State University, Mathematics Department, Ames, IA 50011

E-mail address: hliu@iastate.edu

UCla, Mathematics Department, Los Angeles, CA 90095

E-mail address: ralston@math.ucla.edu 